\title{
PimM, a PAS domain positive regulator of pimaricin biosynthesis in Streptomyces natalensis
}

\author{
Correspondence \\ Jesús F. Aparicio \\ jesus.aparicio@unileon.es
}

Received 17 April 2007

Revised 22 May 2007

Accepted 29 May 2007

\author{
Nuria Antón, ${ }^{1,2}$ Javier Santos-Aberturas, ${ }^{1,2}$ Marta V. Mendes, ${ }^{1} \dagger$ \\ Susana M. Guerra, ${ }^{1,2}$ Juan F. Martín ${ }^{1,2}$ and Jesús F. Aparicio ${ }^{1,2}$ \\ ${ }^{1}$ Institute of Biotechnology INBIOTEC, Parque Científico de León, Avda. Real no 1, 24006 León, \\ Spain \\ ${ }^{2}$ Area of Microbiology, Faculty of Biology, University of León, 24071 León, Spain
}

\begin{abstract}
Sequencing of the DNA region on the left fringe of the pimaricin gene cluster revealed the presence of a $579 \mathrm{bp}$ gene, pimM, whose deduced product (192 aa) was found to have amino acid sequence homology with bacterial regulatory proteins. Database comparisons revealed that PimM combines an N-terminal PAS domain with a C-terminal helix-turn-helix (HTH) motif of the LuxR type. Gene replacement of pimM from the Streptomyces natalensis chromosome with a mutant version lacking the HTH DNA-binding domain resulted in complete loss of pimaricin production, suggesting that PimM is a positive regulator of pimaricin biosynthesis.

Complementation of the $\triangle$ pimM mutant with a single copy of pimM integrated into the chromosome restored pimaricin production. The insertion of a single copy of pimM, with its own promoter, into the $S$. natalensis wild-type strain boosted pimaricin production. Gene expression analyses in S. natalensis wild-type and $\triangle$ pimM by reverse transcriptase PCR (RT-PCR) of the pimaricin gene cluster revealed the targets for the PimM regulatory protein. According to these analyses, the genes responsible for initiation and first elongation cycles of polyketide chain extension are among the major targets for regulation. Other pim genes are differentially affected. Interestingly, our results indicate that PimM plays its regulatory role independently of PimR, the first pathway-specific regulator of pimaricin biosynthesis.
\end{abstract}

\section{INTRODUCTION}

Bacteria belonging to the genus Streptomyces have attracted great interest due to their well known ability to produce a variety of antibiotics and other secondary metabolites. Production of these compounds is regulated in response to nutritional status alteration and a variety of environmental conditions, and hence occurs in a growth-phase-dependent manner and is usually accompanied by morphological differentiation (Martín et al., 2000; Bibb, 2005).

Control of secondary metabolite production is a complex process involving multiple levels of regulation. The highest levels include genes that exert a pleiotropic control over one or more aspects of secondary metabolism, such as antibiotic production or morphological differentiation (for reviews see Champness \& Chater, 1994; Martín, 2004). The lowest level, however, is composed of regulatory genes that only affect a single antibiotic biosynthetic pathway. These

tPresent address: Cellular and Applied Microbiology, IBMC, Institute for Molecular and Cell Biology, R. do Campo Alegre, 823-4150-180 Porto, Portugal.

Abbreviation: HTH, helix-turn-helix.

The GenBank/EMBL/DDBJ accession number for the nucleotide sequence reported in this study is AM493721. pathway-specific regulatory genes are usually found within the respective antibiotic biosynthesis gene cluster, a feature that has greatly facilitated their study. Recently, this scenario has been complicated by the finding of pathwayspecific regulators that can alter the expression of other pathways, and also modulate the effects of regulators that act more globally (Huang et al., 2005).

Regulators often sense chemicals in the environment and respond to changes in their concentration. The most well known example is constituted by the two-component systems involved in phosphorelay signal transduction (Hoch, 2000; West \& Stock, 2001; Foussard et al., 2001). In these systems, a sensory protein phosphorylates a response-regulator in response to an extracellular stimulus (such proteins are usually membrane proteins), and the latter then binds to specific promoter sequences in the DNA, activating or repressing transcription. Two-component regulatory systems are very abundant in Streptomyces species and serve as sensors and transducers of a variety of nutritional and environmental signals (Sola-Landa et al., 2003, 2005; Hutchings et al., 2004; Mendes et al., 2007a).

The PAS domain is a small regulatory module represented in proteins of all kingdoms of life. It is a signal module that monitors changes in light, redox potential, oxygen, overall 
energy level of a cell, and small ligands (Taylor \& Zhulin, 1999). PAS domains were first found in eukaryotes, and were named after homology to the Drosophila period protein (Per), the aryl hydrocarbon receptor nuclear translocator protein (ARNT) and the Drosophila single-minded protein (Sim). Unlike most other sensors, proteins containing PAS domains are located in the cytosol, and therefore they detect internal signals, but they can also sense environmental factors that cross the cell membrane. The majority of prokaryotic PAS domains function as sensor modules of sensor kinases of two-component systems (Taylor \& Zhulin, 1999).

Pimaricin is a tetraene macrolide antifungal antibiotic produced by Streptomyces natalensis. As a polyene, its antifungal activity lies in its interaction with membrane sterols, thus causing the alteration of membrane structure and leading to the leakage of cellular materials (Aparicio et al., 2004). As for other macrocyclic polyketides, pimaricin is synthesized by the action of so-called type I modular polyketide synthases (Aparicio et al., 2003), and its biosynthetic gene cluster has been characterized (Aparicio et al., 1999, 2000; Mendes et al., 2001, 2005, 2007b; Antón et al., 2004).

Here, we describe the cloning, sequencing and detailed characterization of a novel class of PAS domain-containing regulator in $S$. natalensis which does not belong to a twocomponent system, and demonstrate its role as a transcriptional activator for pimaricin biosynthesis in this bacterium.

\section{METHODS}

Bacterial strains, cloning vectors and cultivation. S. natalensis ATCC 27448 was routinely grown in YEME medium (Kieser et al., 2000) without sucrose. Sporulation was achieved in TBO medium (Aparicio et al., 1999). For pimaricin production, the strain was grown in YEME without sucrose. The same media were supplemented with thiostrepton when used for S. natalensis 40D9-1 growth and/or metabolite production. Escherichia coli strain XL1-Blue MR (Stratagene) was used as a host for plasmid subcloning in plasmids pBluescript (Stratagene), pUC18 and pUC19. E. coli ET12567 (pUZ8002) was used as donor in intergeneric conjugations. Candida utilis (syn. Pichia jadinii) CECT 1061 was used for bioassay experiments. Phage KC515 ( ${ }^{+}$attP::tsr::vph), a $\phi \mathrm{C} 31-$ derived phage (Rodicio et al., 1985), was used for gene replacement experiments. Streptomyces lividans JII 1326 (Chater et al., 1981) served as a host for phage propagation and transfection. Infection with $\phi 40 \mathrm{D} 9$ (the KC515 recombinant derivative used for gene replacement) was carried out on R5 medium (Kieser et al., 2000). Standard conditions for culture of Streptomyces species and isolation of phages were as described by Kieser et al. (2000).

Genetic procedures. Standard genetic techniques with E. coli and in vitro DNA manipulations were as described by Sambrook \& Russell (2001). Recombinant DNA techniques in Streptomyces species and isolation of Streptomyces total and phage DNA were performed as described by Kieser et al. (2000). Southern hybridization was carried out with probes labelled with digoxigenin by using the DIG DNA labelling kit (Roche Biochemicals). Intergeneric conjugation between E. coli ET12567(pUZ8002) and S. natalensis was performed as described by Enríquez et al. (2006).

DNA sequencing and analysis. Sequencing templates were obtained by random subcloning of fragments generated by controlled partial HaeIII digestions. DNA sequencing was accomplished by the dideoxynucleotide chain-termination method using the Perkin Elmer AmpliTaq Gold Big Dye-Terminator Sequencing System on doublestranded DNA templates with an Applied Biosystems 310 sequencer. Each nucleotide was sequenced a minimum of three times on both strands. Alignment of sequence contigs was performed using the DNASTAR program SEQMAN. DNA and protein sequences were analysed with the NCBI World Wide Web BLAST server.

Construction of a pimM mutant. A $4083 \mathrm{bp} \mathrm{AccI}$ fragment encompassing the entire pimM gene, and part of the pimR gene (Fig. 1), was cloned into an AccI-cut pUC19 vector to yield pMVM500. This plasmid was then used as a source of DNA for both sequencing of pimM and for obtaining the DNA fragment used for gene replacement.

Using plasmid pMVM500 as a template, a $1.4 \mathrm{~kb}$ DNA fragment containing the whole of $p i m M$ was amplified by PCR with primers DWM (5' -AAAGCGGCAGATCTTCGGCAAGGATTC- $3^{\prime}$ ) and UPM (5'-AGCAGAGATCTGGACGGCGACGGCACTC-3') (underlined sequences indicate $B g l I I$ restriction sites). The PCR product was digested with BglII and ligated into a BamHI-cut pUC19 vector to yield pMVM600.

The pimM gene was disrupted by KC515 phage-mediated gene replacement as follows. Plasmid pMVM600 was digested with ApaI and religated to yield pMVM620. This treatment eliminates a $387 \mathrm{bp}$ ApaI fragment that encodes the C-terminal end (123 aa) of PimM which contains the helix-turn-helix (HTH) domain for DNA binding (Stevens et al., 1994), and results in a mutant pimM gene truncated beyond the new ApaI site. A $1 \mathrm{~kb}$ SacI-PstI fragment containing the mutant pimM sequence was cloned into the same sites of KC515 (Kieser et al., 2000). Transfection of Streptomyces lividans protoplasts (Kieser et al., 2000) resulted in a number of phage plaques that were screened by Southern hybridization for the presence of pimM-derived sequences. One of the recombinants, $\phi 40 \mathrm{D} 9$, was selected and used to infect S. natalensis, thus allowing selection for lysogen formation. Lysogens were selected by thiostrepton resistance on R5 medium. Gene replacement was sought by repeated rounds of non-selective growth in liquid YEME medium without sucrose, and the loss of the phage was confirmed by genomic Southern hybridization.

Construction of plasmids for gene complementation and gene dosage increase. In order to complement the pimM replacement mutant, a $1 \mathrm{~kb}$ DNA fragment containing the entire pimM gene, including its own promoter, was amplified by PCR with primers PMD (5'-TCCTGGATCCGCCCTGTGCCCGCTCACTTCACGAAGTCG-3') and PMR (5'-GGTTGGATCCTTGCGGTCGGTGGTGCGGGCATTACGG-3') (the underlined sequences indicate BamHI restriction sites). The PCR product was digested with BamHI and ligated into BamHI-cut pSET152 (Am ${ }^{\mathrm{R}}$, pUC18 replicon, $\phi \mathrm{C} 31$ attP; Bierman et al., 1992) to yield pSETpimM. This plasmid was then transferred by conjugation from E. coli ET12567(pUZ8002) to the $S$. natalensis $\Delta$ PimM mutant as described by Enríquez et al. (2006).

Isolation of total RNA. S. natalensis ATCC 27448 and S. natalensis $\triangle$ pimM were grown for $48 \mathrm{~h}$ in YEME medium without sucrose (stationary phase). The cultures were then mixed with one volume $40 \%(\mathrm{v} / \mathrm{v})$ glycerol, and mycelia were harvested by centrifugation and immediately frozen by immersion in liquid nitrogen. Frozen mycelium was then broken by shearing in a mortar, and the frozen lysate was added to buffer RLT (Qiagen) in the presence of $1.5 \%(\mathrm{v} / \mathrm{v})$ $\beta$-mercaptoethanol. RNeasy Mini Spin columns were used for RNA isolation according to manufacturer's instructions. RNA preparations were treated with DNase I (Promega) to eliminate possible chromosomal DNA contamination.

Gene expression analysis by reverse transcriptase PCR (RTPCR). Transcription was studied by using the SuperScript One-Step 

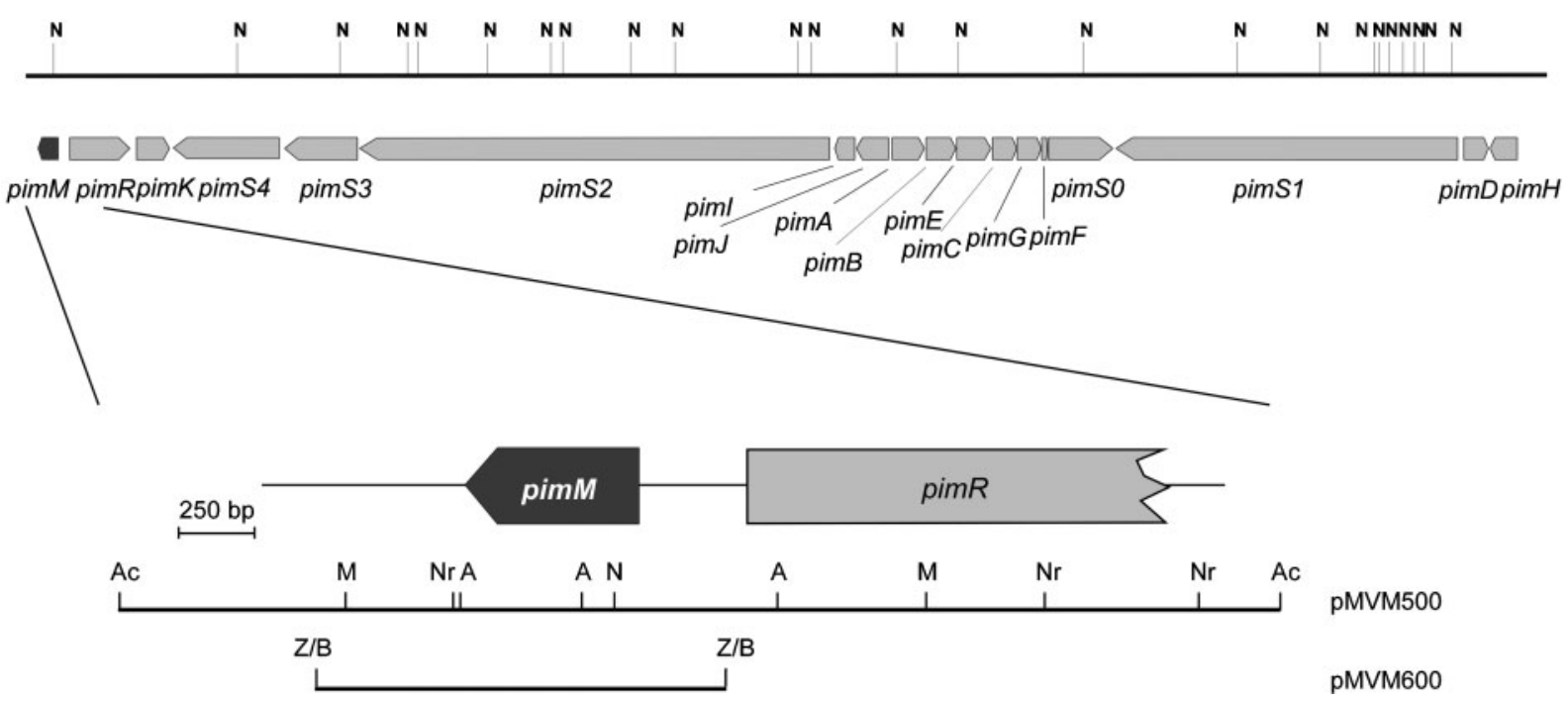

Fig. 1. Pimaricin biosynthetic gene cluster. The left fringe of the gene cluster is indicated in more detail, and includes pimM (in dark grey), and the $5^{\prime}$ end of the transcriptional activator-encoding gene for pimaricin biosynthesis (pimR; Antón et al., 2004). Arrowed boxes indicate the direction of transcription. A, Apal; Ac, Accl; B, BamHI; M, Mlul; N, Notl; Nr, Nrul; Z, Bg/ll. The inserts used for vector construction are indicated at the bottom.

RT-PCR system with Platinum Taq DNA polymerase (Invitrogen), using $5 \mathrm{ng}$ total RNA as template. Conditions were as follows: first strand cDNA synthesis, $45{ }^{\circ} \mathrm{C}$ for $40 \mathrm{~min}$ followed by heating at $94{ }^{\circ} \mathrm{C}$ for $2 \mathrm{~min}$; amplification, 28 or 30 cycles of $98{ }^{\circ} \mathrm{C}$ for $15 \mathrm{~s}, 60$ $70{ }^{\circ} \mathrm{C}$ (depending on the set of primers used) for $30 \mathrm{~s}$, and $72{ }^{\circ} \mathrm{C}$ for 1 min. Primers (18- to 24-mers; Antón et al., 2004) were designed to generate PCR products of approximately $500 \mathrm{bp}$. The primers used for the detection of the pimM transcripts were PIMMS $\left(5^{\prime}\right.$ TGGCGAGCCTTGATAGAACATTGAC-3') and PIMMAS (5'-CCGGTGGCGCAGGGTGAAC- $\left.3^{\prime}\right)$. The $5^{\prime}$ end of PIMMAS is located $84 \mathrm{nt}$ downstream from the pimM TGA stop codon. PCR products using these primers were $661 \mathrm{bp}$ for the wild-type strain, and $274 \mathrm{bp}$ for the mutant. The antisense primer used for the detection of pimA transcripts (PIMAAS) differs from that used by Antón et al. (2004), and its sequence is $5^{\prime}$-GACCAGCAGCACCAGGGGGATGAC- ${ }^{\prime}$. The primers used for the analysis of pim $A B$ mRNA stability were PIMABS (5'-GCTGCTGCGCACCAGTACGACG-3') and PIMABAS (5'-GCTGAGGGTCTGCTGGAGGGTCTGCTGGAGGTTGTC-3'). Negative controls were carried out with each set of primers and Platinum Taq DNA polymerase to confirm the absence of contaminating DNA in the RNA preparations. The identity of each amplified product was corroborated by direct sequencing of the PCR product.

Assay of pimaricin production. Spectrophotometric determination of pimaricin was performed as described elsewhere (Recio et al., 2004). HPLC analysis of this antifungal was carried out as described by Recio et al. (2006). The fungicidal activity of pimaricin was tested by bioassay using C. utilis CECT 1061 as test organism.

\section{RESULTS}

\section{Cloning of pimM}

pimM was identified by genomic walking using an $S$. natalensis ATCC 27448 cosmid library (Aparicio et al., 1999) and DNA segments from pimR (which encodes a pathway-specific regulatory gene for pimaricin production, on the left end of the pimaricin gene cluster; Antón et al., 2004). The gene was sequenced from plasmid pMVM500 (see Methods) and turned out to be separated by $396 \mathrm{bp}$ from the $5^{\prime}$ end of $\operatorname{pim} R$, orientated in a divergent fashion (Fig. 1). The initiating ATG codon of pimM is preceded by the sequence AGGGAG which could act as a ribosomebinding site. pimM is $579 \mathrm{bp}$ long with an overall codon usage pattern in good agreement with that of typical Streptomyces genes; however, it contains a few codons that are rare in such a $\mathrm{G}+\mathrm{C}$-rich organism. The presence of one TTA codon could be of particular interest, since its involvement in the regulation of differentiation and secondary metabolism in Streptomyces has been proposed in other Actinomycetes (Leskiw et al., 1991).

\section{In silico analysis of the pimM gene product}

Computer-assisted analysis of the pimM gene product (192 aa with an estimated molecular mass of $20945 \mathrm{Da}$ ) showed a very high sequence identity $(93.8 \%)$ with the whole of protein PTEF of Streptomyces avermitilis, a putative regulatory protein of 232 aa whose gene was found within the pte gene cluster and which is thought to be involved in the biosynthesis of the pentaene filipin (Ikeda et al., 2003). Protein database comparisons revealed three additional counterparts, all encoded by regulatory genes of polyene biosynthetic clusters. These were AmphRIV (68\% identity) (amphotericin; Carmody et al., 2004), NysRIV (66.8\% identity) (Nystatin; Sekurova et al., 2004) and FscRI (67.5\% identity) (FR008/Candicidin; Chen et al., 2003). All of them have a PAS sensor-binding domain at the $\mathrm{N}$ terminus (Taylor \& Zhulin, 1999; Hefti et al., 2004; 
SMART 00091) as well as an HTH motif of the LuxR type at the $\mathrm{C}$ terminus (SMART 00421) (Fig. 2). Interestingly, PimM is $17-49$ aa shorter than its counterparts.

When we analysed sequence identity at the DNA level, we found a strikingly high similarity between pimM and pteF (93.4\% identity along the whole length of pimM). This sequence identity is extremely high, and indicates that both regulatory genes probably have a common evolutionary origin. Curiously, the identity at the DNA level continued beyond the pimM ATG start codon, in the non-coding region upstream of ATG. A reassignment of the start codon according to the $p t e F$ coding sequence would yield a protein of identical size to PteF. However, the presence of a TGA triplet in the pimM-elongated sequence, $69 \mathrm{nt}$ upstream from the pimM ATG start codon, removes such possibility.

\section{Gene replacement of pimM}

The involvement of pimM in pimaricin biosynthesis was tested by gene replacement. We used phage KC515, an attP-defective $\phi \mathrm{C} 31$ derivative (Rodicio et al., 1985), to introduce DNA into $S$. natalensis. The recombinant phage used for pimM inactivation, $\phi 40 \mathrm{D} 9$, was constructed as described in Methods and used to infect S. natalensis to obtain lysogens. Because phage KC515 and its derivative lack attP, they can only form lysogens by homologous recombination into the chromosome (Fig. 3a).

Nine lysogens of $S$. natalensis were obtained by selection for thiostrepton resistance. One of these mutants was randomly selected and named S. natalensis 40D9-1. The identity of the mutant was confirmed by Southern hybridization (not shown). This mutant was then used to isolate thiostrepton-sensitive derivatives that had undergone a second recombination event deleting the integrated phage. These thiostrepton-sensitive isolates were obtained after 11 rounds of non-selective growth in YEME medium. Of the three colonies isolated, two were found to have reverted to the wild-type, while the other one harboured the desired change.

This mutant, where pim $M$ had been replaced by a mutated version of it lacking the C-terminal HTH binding site, was named S. natalensis $\Delta$ pimM. The truncated protein is devoid of DNA-binding ability, and its function as a regulator should therefore be lost. Chromosomal DNAs isolated from S. natalensis ATCC 27448 and mutant $\Delta p i m M$ digested with $M l u \mathrm{I}$ were probed with a $431 \mathrm{bp}$

(a)

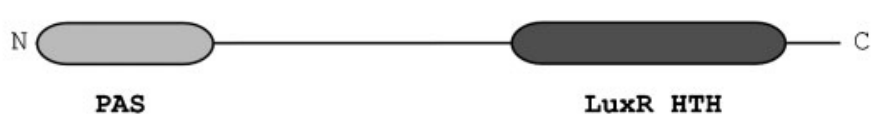

(b)

\begin{tabular}{|c|c|c|}
\hline AmphRIV & 50 & LARLDQALTIQQASEGFFWQFGGSSAELCGRTFSDLVHPSVQQPLMRQFSGLIE \\
\hline NysRIV & 18 & MASLDRDLTIKHANQEFRRRFDDSAGDVCGRSFRDLMHPSVQQPLMRQ̋FSRLIE \\
\hline EscRI & 30 & VACLDPSLTIQQVNQEFERRFGGSSSELCGSRFCDLVHPSVQQPLMHQFARMLD \\
\hline PteF & 41 & MASLDKTLTIQQANQEFFRQFNGSSEDICGRNFRDVVHPSVQQPLVRQFSNLLE \\
\hline PimM & 1 & MASLDRTLTIQQANQEFFRQFNGSSEDICGRNFRDVVHPSVQQPLVRQFSNLLE \\
\hline & & 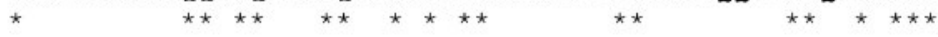 \\
\hline
\end{tabular}

(c)

\begin{tabular}{|c|c|c|c|}
\hline & & Helix & Helix \\
\hline GerE & 009 & \multicolumn{2}{|c|}{ KPSLTKREREVFELLVQDKTTKEIASELFISEKTVRNHISNAMQKLGVKGRSQAVVELLRMG } \\
\hline AmphRIV & 164 & \multicolumn{2}{|c|}{ KKILSPIDARILEGIASGLSTIPLASRLHLSRQGIEYHVTCLLRKLRVPNRAALVSRAYSMG } \\
\hline NysRIV & 132 & \multicolumn{2}{|c|}{ KKELTEIDARILEGIAAGLSTIPLASRLYLSRQGVEYHVTGLLRKLRVPNRAALVSRAYSMG } \\
\hline FSCRI & 144 & \multicolumn{2}{|c|}{ KKLISEIDARI LEGIAAGVSTIPLASRLYLARQGVEYHVTGLLRKLKVPNRAALVSRAYSMG } \\
\hline PteF & 154 & \multicolumn{2}{|c|}{ KKILSTMDARILEGIAAGVSTVPLAASLYLSRQGVEYHVTCLLRKLKVSNRAALVSRAYSLG } \\
\hline PimM & 114 & \multicolumn{2}{|c|}{ KKILSAMDARILEGIAAGVSTVPIAARLYLSRQGVEYHVTCLLRKLKVPNRAALVSRAYSMG } \\
\hline & & $\star \star \star \quad * \star$ & $\star \star$ \\
\hline
\end{tabular}

Fig. 2. Domain structure and amino acid sequence alignments of parts of the PimM protein. (a) Predicted domain structure of PimM. PAS, PAS sensory domain (SMART 00091); LuxR HTH, DNA-binding domain of the LuxR type (SMART 00421). (b) Sequence comparison of the N-terminal PAS domains of PimM and other regulators of polyene biosynthetic gene clusters. AmphRIV, NysRIV, FscRI and PteF are transcriptional activators of amphotericin, nystatin, candicidin and filipin clusters, respectively. (c) Sequence alignment of the C-terminal LuxR-type HTH DNA-binding domains of PimM orthologues and GerE. GerE is the transcriptional regulator of spore formation in Bacillus subtilis. The horizontal bars at the top represent the location of the HTH motif in the proposed DNA-binding region. Numbers indicate amino acid residues from the $\mathrm{N}$ terminus of the protein. Identical residues are shown in bold. An asterisk $\left(^{*}\right)$ signifies a conservative substitution. 
(a)

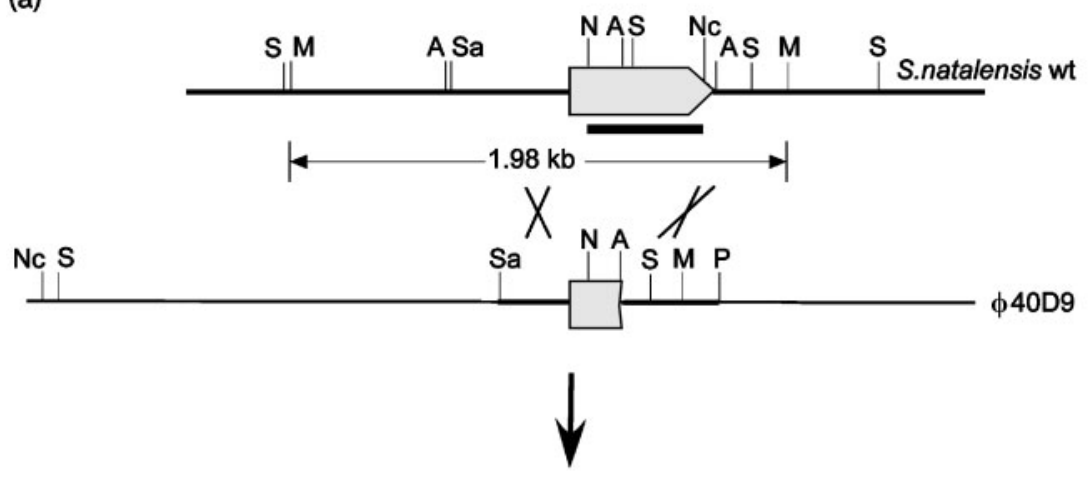

(b)

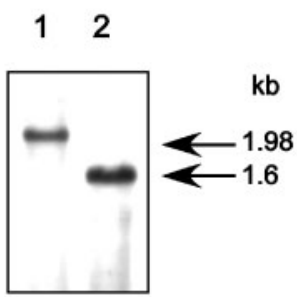

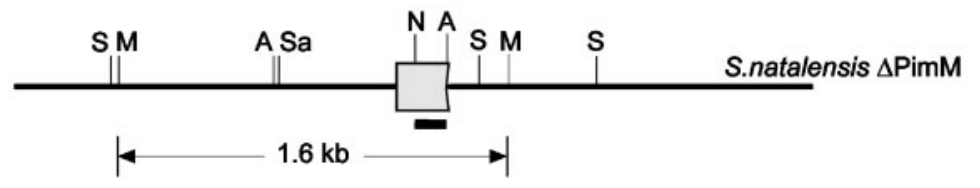

Fig. 3. Gene replacement of pimM. (a) Predicted restriction enzyme polymorphism caused by gene replacement. The Mlul restriction pattern before and after replacement is shown. The probe is indicated by thick lines. The fragment used for gene disruption is derived from pMVM620 (see text for details). A, Apal; M, Mlul; N, Notl; Nc, Ncol; S, Sacll; Sa; Sacl. (b) Southern hybridization of Mlul-digested chromosomal DNA of wild-type (lane 1) and $\Delta$ pimM (lane 2) strains.

NotI-NcoI fragment covering most of pimM (Fig. 3a). A hybridizing band of $1.98 \mathrm{~kb}$ was found for the wild-type as expected (Fig. 3b), whereas in the mutant, the hybridizing band was $1.6 \mathrm{~kb}$ (Fig. 3b), indicating that a double crossover event had occurred. The observed hybridizing bands corresponded exactly to those expected, according to the integration process depicted in Fig. 3.

The new strain $S$. natalensis $\Delta$ pimM had growth and morphological characteristics identical to those of $S$. natalensis wild-type when grown on solid or liquid media, suggesting that PimM has no role in bacterial growth or differentiation. The spore counts of both strains were similar after growth for 9 days at $30{ }^{\circ} \mathrm{C}$ on TBO plates. The spores of both strains were serially diluted and plated on minimal medium. Both strains grew well in minimal medium, showing an identical growth curve, which indicates that genes involved in amino acid biosynthesis were not affected. Also, no differences in pigment production were observed in the mutant, suggesting that the regulator might be specific for pimaricin biosynthesis.

\section{Inactivation of pimM blocked pimaricin biosynthesis and gene complementation restored antifungal production}

The fermentation broth produced by the mutant strain generated by phage-mediated gene replacement, S. natalensis $\Delta$ pimM, was extracted with butanol and analysed for the presence of pimaricin. Both the microbiological bioassay against $C$. utilis and HPLC indicated that no pimaricin was being produced by the mutant strain $\Delta$ pimM (Fig. 4). This result indicated that PimM was a positive regulator for pimaricin biosynthesis, which together with its high sequence similarity with transcriptional activators of polyene biosynthesis (see above) raised the question of which gene/genes were the potential target of PimM activity.

To confirm that the disruption of pimM was directly responsible for the abolition of pimaricin production, we complemented the pimM-disrupted mutant with pimM. A DNA fragment containing pimM plus its putative promoter region was inserted into the integrative vector pSET152 (Bierman et al., 1992), giving rise to pSETpimM (see Methods). The recent development of a method for intergeneric gene transfer from E. coli to $S$. natalensis by conjugation (Enríquez et al., 2006) enabled its use for the introduction of pSETpimM into the pimM-disrupted mutant. The plasmid was thus transferred from E. coli ET12567(pUZ8002) to S. natalensis $\Delta$ pimM. Introduction of pSETpimM restored pimaricin biosynthesis to wild-type levels. These results were fully consistent with those obtained upon replacement of the pimM gene, and confirmed the involvement of PimM in pimaricin biosynthesis.

\section{Duplication of gene dosage increases pimaricin production}

When we introduced pSETpimM into S. natalensis wildtype, whereas no significant change in the growth curve in flask cultures was observed, a substantial increase in the specific production of pimaricin was detected (Fig. 5), thus suggesting that PimM availability does constitute a bottleneck in the biosynthesis of the antifungal. The increase in 


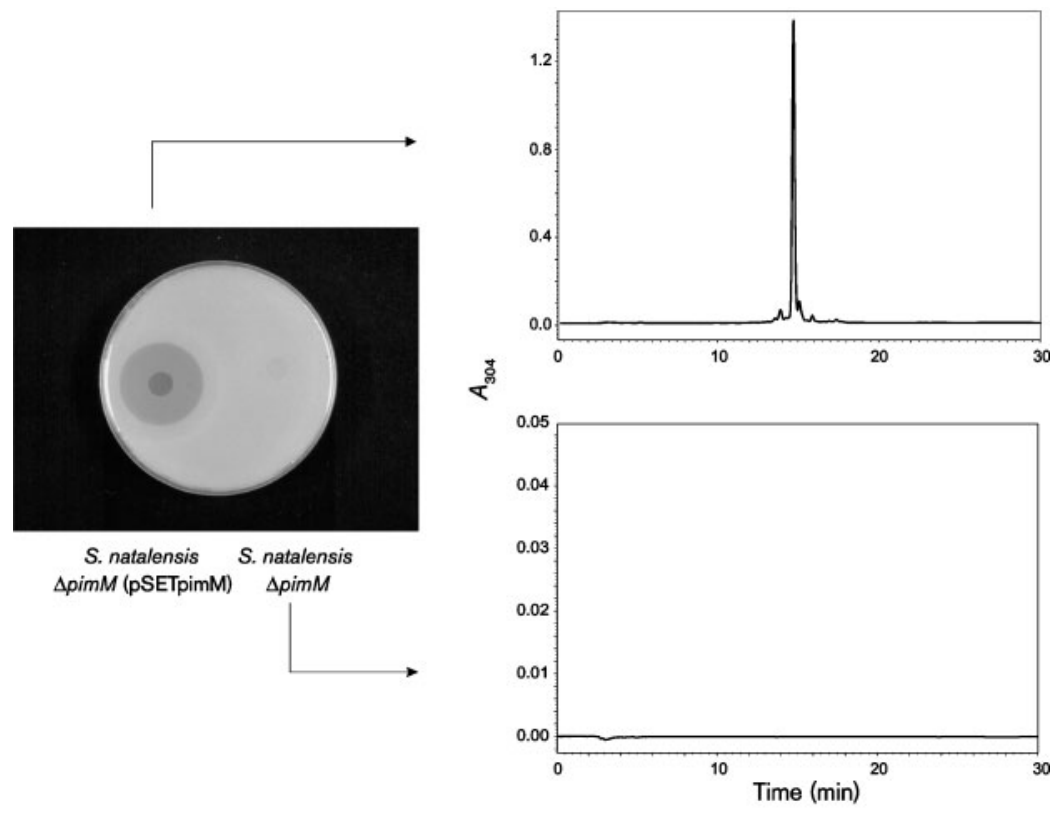

Fig. 4. Replacement of PimM blocks pimaricin production, and gene complementation restores antifungal biosynthesis. Comparison of HPLC analyses of butanol-extracted broths from $S$. natalensis $\triangle$ pimM (bottom) and $S$. natalensis $\triangle$ pimM transformed with pSETpimM (top). Detection was carried out at $A_{304}$. Cells were grown for $48 \mathrm{~h}$ with shaking at 300 r.p.m. and at $30{ }^{\circ} \mathrm{C}$ in YEME medium. Bioassays for pimaricin production are included on the left using C. utilis as test organism.

pimaricin production ranged between 2.4-fold after $48 \mathrm{~h}$ of growth to 1.5 -fold after $96 \mathrm{~h}$, and taking into consideration the similar growth curves of the strain with an extra copy of pimM and the parental strain, one can conclude that it is mostly derived from a higher volumetric production in the strain with a double gene dosage, which reached $1.48 \mathrm{~g} \mathrm{l}^{-1}$ at its maximum $(72 \mathrm{~h})$.

\section{Transcriptional control of pimaricin production}

Total RNA was prepared from S. natalensis wild-type and mutant $\triangle$ pimM after growth for $48 \mathrm{~h}$ (when pimaricin is actively produced; Mendes et al., 2001) and used as template for gene expression analysis by RT-PCR. Primers for RT-PCR were specific to sequences within pim genes (see Methods) and were designed to produce cDNAs of approximately $500 \mathrm{bp}$. A primer pair designed to amplify a cDNA of the lysA gene (encoding diaminopimelate decarboxylase) was used as an internal control. Transcripts were analysed from the 18 genes of the pim cluster, including pimM, after 28 PCR cycles. In the case of pimM, transcripts were analysed using a sense primer located before the deletion (PIMMS), and an antisense primer located after the stop codon (PIMMAS) (see Methods). Whenever 28 cycles did not yield a product, analysis was repeated at 30 cycles. These analyses were carried out at least three times for each primer pair.

All 18 genes were transcribed at $48 \mathrm{~h}$ in S. natalensis wildtype; however, when we analysed the transcription pattern in $S$. natalensis $\triangle p i m M$, we found no transcripts for the genes pimD, pimS1, pimSO, pimF, pimG, pimC and pimB (Fig. 6a) even after increasing the number of cycles to 30 (Fig. 6a), indicating that the promoters controlling the expression of these genes are likely targets, directly or indirectly, for PimM regulatory control. Similarly, no transcripts were found for the genes pimJ and pimI (Fig. 6a), but in these cases increasing the number of cycles allowed the detection of a very small amount of their transcripts (Fig. 6a), thus suggesting that the mutant retains some transcription of these pim genes albeit at very low levels. Transcription was also substantially reduced for pimK and pimA, and for the polyketide synthase-encoding genes pimS2, pimS3 and pimS4, although in these cases, the transcripts were clearly detected after 28 cycles of amplification (Fig. 6a). Interestingly, no difference in the transcription pattern was observed for the genes $p i m R$, pimE and pimH (Fig. 6a), thus suggesting that pimM and the first regulatory gene for pimaricin biosynthesis pimR (Antón et al., 2004) function independently of each other (see Discussion). A similar transcription pattern was also observed for pimM, although with different size RT-PCR amplification products for the wild-type and the mutant; given that primers are located at both sides of the deletion, this indicates that transcription proceeded unabated across the site of the in-frame deletion in pimM. This result indicates that the gene replacement does not have a polar effect on the transcription of genes located downstream from pimM, and also that PimM is not auto-regulated. The transcription pattern of $l y s A$ (a primary metabolism gene) was comparable in S. natalensis $\Delta$ pimM and in the parental strain (Fig. 6a), thus validating the results described above.

Analysis of the transcription pattern of the pimM gene in the S. natalensis $\triangle p i m R$ mutant previously obtained (Antón et al., 2004) revealed that pimM is not regulated by PimR (Fig. 6b).

The strict control of PimM on the transcription of pimSO, which encodes the polyketide synthase responsible for starting polyketide chain construction (Aparicio et al., 

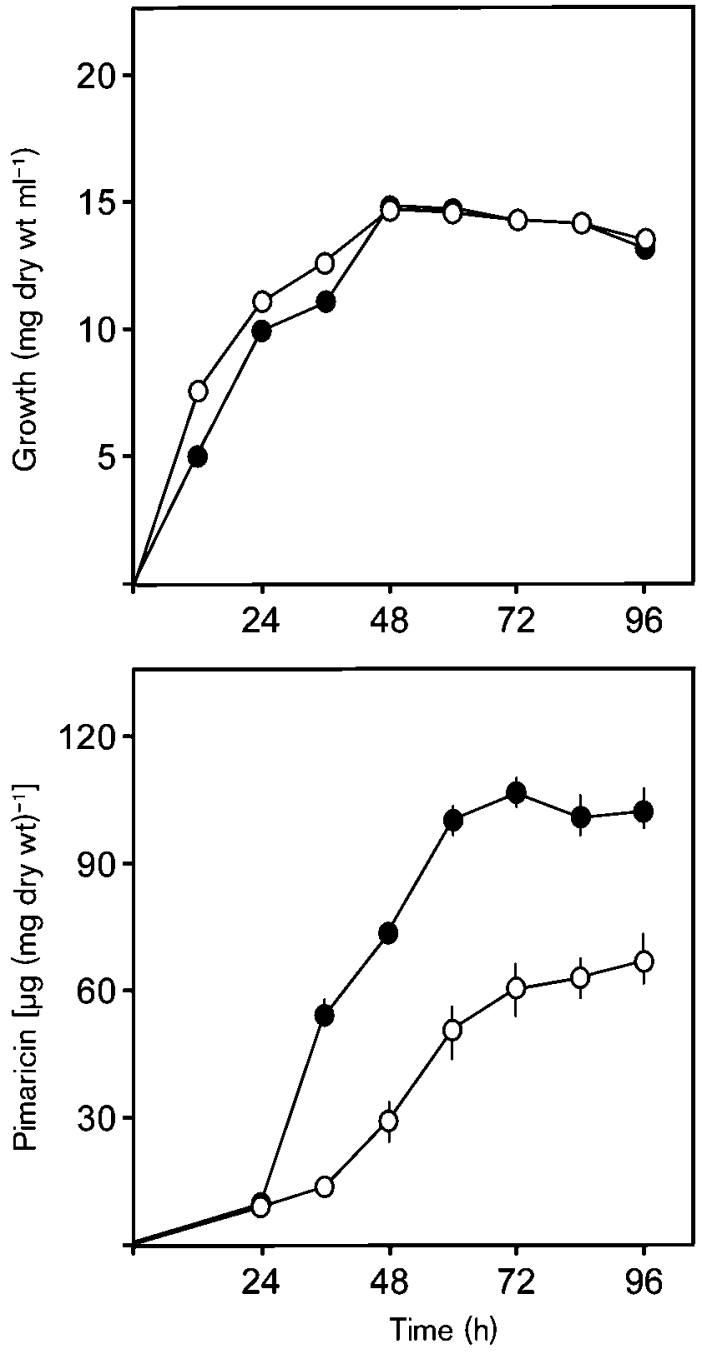

Fig. 5. Increase in pimM gene dosage increases pimaricin production. Specific production of pimaricin [expressed as $\mu \mathrm{g}$ pimaricin (mg dry wt) ${ }^{-1}$ ] in YEME medium without sucrose (lower panel). Open circles, production by the wild-type strain; solid circles, production observed in the strain with an extra copy of pimM. Data are the means of three duplicate flasks. Vertical bars indicate the SD values. Growth curves are shown in the upper panel.

2000), is sufficient to explain the lack of pimaricin production in the mutant. pimSO most probably forms a multicistronic operon together with pim C, pimG and pimF (Antón et al., 2004), and hence all of them are controlled coordinately. The genes pimD and pimS1 are transcribed as monocistronic units as can be deduced from their chromosomal arrangement in a divergent fashion (Fig. 6a). The lack of pimS1 transcription in the mutant strain could also account for the lack of pimaricin production in the mutant.

The differential control of PimM on the transcription of pimJ and pimI when compared with that of pimS2, pimS3 and pimS4 was somehow unexpected because, due to the absence of apparent transcriptional terminators in the short intergenic regions between them, all these genes were thought to form an operon resulting in a transcript of more than $42 \mathrm{~kb}$ (Aparicio et al., 2000). These results now could indicate that the above-indicated genes are actually transcribed from at least two different transcriptional units, namely pimJI and pimS2S3S4. However, in the absence of evidence indicating that all the transcripts are equally stable it is also possible that the multicistronic transcript could be processed and subject to different rates of RNA degradation.

Interestingly, pimA and pimB showed a different transcription pattern. While transcription of pimA was reduced in $S$. natalensis $\triangle p i m M$ when compared to the parental strain, no transcripts could be detected for pimB in the mutant (Fig. 6a), even after increasing the number of cycles to 30 (Fig. 6a). Since both genes are thought be translationally coupled, given that their coding sequences overlap ( start codon is located $23 \mathrm{bp}$ upstream from the pimA stop codon; Aparicio et al., 2000), the most likely explanation for this result is the fast degradation of pimB transcripts. To check this possibility we designed primers to give an amplification band covering the $3^{\prime}$ end (last $142 \mathrm{nt}$ ) of pimA and the $5^{\prime}$ end (first $465 \mathrm{nt}$ ) of pimB (Fig. 7). The result of the RT-PCR analysis showed a band of similar intensity to that obtained with pimA primers, thus indicating that the $3^{\prime}$ end of the $\operatorname{pim} A B$ mRNA was particularly prone to degradation in the mutant.

\section{DISCUSSION}

Two distinct regulators of pimaricin biosynthesis are encoded by genes located in the pim cluster, pimR and pimM. PimR is the archetype of a novel class of regulators that combines an $\mathrm{N}$-terminal domain corresponding to the SARP family of transcriptional activators, a central domain with similarity to the NTP-binding motif of the LuxR family of DNA-binding proteins, and a C-terminal domain that resembles guanylate cyclases. Gene disruption of $\operatorname{pim} R$ totally abrogated pimE transcription, and also reduced the transcription of all the key enzyme-encoding genes for pimaricinolide construction to very low levels, thus blocking pimaricin production completely (Antón et al., 2004).

Sequencing of the left-hand side of the pimaricin gene cluster revealed the presence of a gene, pimM, which could play a role as a regulator for pimaricin biosynthesis in $S$. natalensis. Computer-assisted analysis of PimM revealed that it has an N-terminal region strikingly similar to PAS sensory domains (Taylor \& Zhulin, 1999; Hefti et al., 2004) and a C-terminal region with a LuxR-type HTH motif for DNA binding. The presence of a PAS-like domain within PimM suggests that this protein could respond to the energy levels in the cell (Ponting \& Aravind, 1997), while the HTH motif suggests the ability of PimM to bind DNA 
(a)
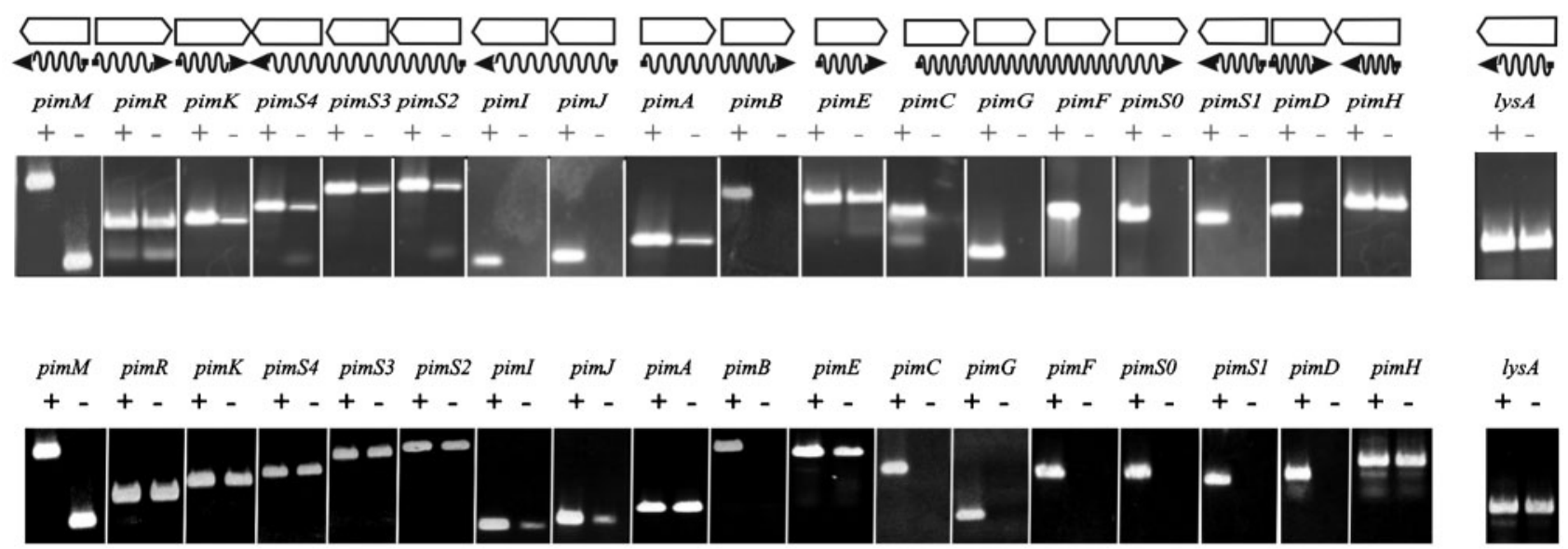

(b)

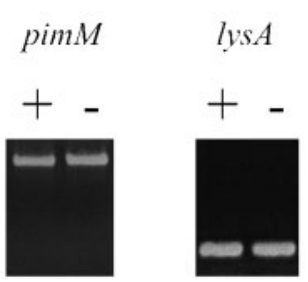

Fig. 6. Gene expression analysis of the pimaricin gene cluster by RT-PCR. (a) Analysis was carried out on S. natalensis wildtype $(+)$ and $\Delta$ pimM $(-)$ strains as indicated in Methods after 28 (top panel) and 30 (bottom panel) PCR amplification cycles. The identity of each amplified product was corroborated by direct sequencing. The absence of contaminating DNA in the RNA samples was assessed by PCR. Due to its small size, transcription of pimF was assessed by using a reverse primer located within the coding sequence of the gene located downstream from it (i.e. pimSO). Transcription of pimM or its mutated version was assessed by using a reverse primer located after the deletion, downstream from the stop codon (note the different size of the amplification products). Transcription of the lys $A$ gene (encoding diaminopimelate decarboxylase) located outside the pim cluster was also assessed as an internal control. A diagram with the organization of the genes within the pimaricin cluster and their putative transcripts is also included (the length of the arrows is not proportional to the size of the genes). (b) Comparative expression of the pimM gene in the wild-type S. natalensis $(+)$ and in a previously obtained $\Delta$ pimR mutant (-) (Antón et al., 2004) after 28 PCR amplification cycles.

(Stevens et al., 1994) and thus regulate the expression of pimaricin genes. The absence of pimaricin production upon disruption of the gene by removal of the HTH domain clearly indicates that PimM is an activator of pimaricin biosynthesis. It constitutes the second activator of pimaricin biosynthesis, after PimR (Antón et al., 2004).

Our results indicate that the control of pimaricin biosynthesis exerted by PimM takes place through the specific transcriptional activation of some key enzymeencoding genes for pimaricinolide construction. These include pimSO and pimS1, the genes that encode the polyketide synthases responsible for polyketide chain initiation and the first four elongation cycles of polyketide chain construction (Aparicio et al., 2000). The lack of expression of any of them could explain the lack of pimaricin production in the mutant. PimM also controls the expression of other genes such as pimC (encoding transaminase, putatively involved in the biosynthesis of the mycosamine moiety of pimaricin), pim $G$ (cytochrome P450 mono-oxygenase candidate for the formation of the exocyclic carboxyl group) and pimF (ferredoxin) (Aparicio et al., 2003). This is not surprising, since they are thought to form a multicistronic operon together with pimSo (Antón et al., 2004). The epoxidase-encoding gene pimD (Mendes et al., 2005) is also controlled by PimM. pimD is transcribed as a monocistronic unit whose expression is controlled most probably by a bidirectional promoter, given the divergent orientation of pimD and pimS1.

Besides these major targets for PimM control, other genes display reduced transcription upon gene disruption of pimM. Among these, the most strongly affected by the lack of PimM are pimJ (GDP-mannose dehydratase) and pimI (discrete thioesterase putatively involved in the removal of non-productive decarboxylated extender acyl groups from the polyketide synthase; Kim et al., 2002). Others, like pimS2, pimS3 and pimS4, the remaining three polyketide 


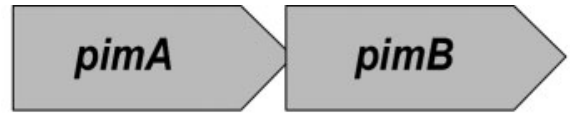

mRNA

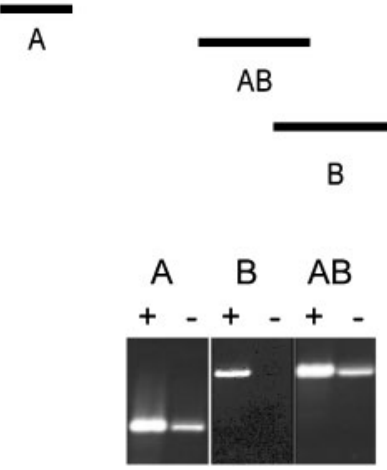

Fig. 7. Gene expression analysis of the pimAB operon by RTPCR. Comparative expression in the wild-type $S$. natalensis (+) and in the $\Delta$ pimM mutant $(-)$ was assessed using primers corresponding to different locations in the mRNA (see text). Expected amplification cDNA bands are indicated for the various primer pairs. The RT-PCR analysis was carried out after 28 PCR amplification cycles.

synthase genes responsible for the formation of the pimaricinolide (Aparicio et al., 2000), pimA and pimB, which encode a putative heterodimer $\mathrm{ABC}$ transporter involved in pimaricin secretion (Aparicio et al., 2003), and $\operatorname{pim} K$, involved in the attachment of the mycosamine moiety during pimaricin biosynthesis (unpublished results), are also affected, although to a reduced extent.

PimM either controls multiple pimaricin biosynthetic promoters directly or activates another hierarchical regulator(s). This does not seem to be the case for pimR, the transcriptional activator of pimaricin biosynthesis first characterized by Antón et al. (2004). PimR, as well as PimM, is not auto-regulated (Antón et al., 2004) and does not control pimM expression (Fig. 6b). Similarly, PimM does not control the expression of $\operatorname{pimR}$, and neither that of pimH (efflux pump) nor pimE (cholesterol oxidase; Mendes et al., 2007b). Since pimE seems to be the main target of PimR (Antón et al., 2004), most probably both regulators belong to different regulatory circuits.

Only NysRIV amongst PimM orthologues has been characterized, in this case by gene disruption and in vivo complementation experiments (Sekurova et al., 2004). According to these studies, nysRIV most probably directly controls the expression of nystatin biosynthetic genes, and that is also the case of pimM for the pim genes. Interestingly, the promoter that drives the expression of $n y s A$, the gene responsible for the initiation of nystatin biosynthesis (Brautaset et al., 2000), seems to be the major target for the NysRIV regulator (Sekurova et al., 2004), as occurs with pimSo (functionally equivalent to nysA) for PimM. Similarly, NysRIV also controls expression from the $n y s \mathrm{H}$ promoter, which drives the expression of the $\mathrm{ABC}$ transporter for nystatin secretion, as seen with the operon pimAB in the case of PimM. All these similarities suggest that both regulators might follow a similar regulatory pattern for the expression of their respective polyenes, and that this pattern could be shared by the homologous regulatory genes found in other polyene biosynthetic gene clusters.

\section{ACKNOWLEDGEMENTS}

This work was supported by Spanish Ministry of Education and Science Grants to J. F. A. (BIO2004-00066 and GEN2003-20245-C0908/NAC). N.A. and J.S.-A. were the recipients of FPU fellowships from the Ministerio de Educación y Ciencia (AP2002-1446 and AP2005-3644, respectively). S. M. G. received a fellowship from the University of León. We thank the excellent technical assistance of J. Merino, B. Martín and A. Casenave.

\section{REFERENCES}

Antón, N., Mendes, M. V., Martín, J. F. \& Aparicio, J. F. (2004). Identification of $\mathrm{PimR}$ as a positive regulator of pimaricin biosynthesis in Streptomyces natalensis. J Bacteriol 186, 2567-2575.

Aparicio, J. F., Colina, A. J., Ceballos, E. \& Martín, J. F. (1999). The biosynthetic gene cluster for the 26-membered ring polyene macrolide pimaricin: a new polyketide synthase organization encoded by two subclusters separated by functionalization genes. $J$ Biol Chem 274, 10133-10139.

Aparicio, J. F., Fouces, R., Mendes, M. V., Olivera, N. \& Martín, J. F. (2000). A complex multienzyme system encoded by five polyketide synthase genes is involved in the biosynthesis of the 26-membered polyene macrolide pimaricin in Streptomyces natalensis. Chem Biol 7, 895-905.

Aparicio, J. F., Caffrey, P., Gil, J. A. \& Zotchev, S. B. (2003). Polyene antibiotic biosynthesis gene clusters. Appl Microbiol Biotechnol 61, 179-188.

Aparicio, J. F., Mendes, M. V., Antón, N., Recio, E. \& Martín, J. F. (2004). Polyene macrolide antibiotic biosynthesis. Curr Med Chem 11, 1645-1656.

Bibb, M. J. (2005). Regulation of secondary metabolism in streptomycetes. Curr Opin Microbiol 8, 208-215.

Bierman, M., Logan, R., O’Brien, K., Seno, E. T., Rao, R. N. \& Schoner, B. E. (1992). Plasmid cloning vectors for the conjugal transfer of DNA from Escherichia coli to Streptomyces spp. Gene 116, 43-49.

Brautaset, T., Sekurova, O. N., Sletta, H., Ellingsen, T. E., Strom, A. R., Valla, S. \& Zotchev, S. B. (2000). Biosynthesis of the polyene antifungal antibiotic nystatin in Streptomyces noursei ATCC 11455: analysis of the gene cluster and deduction of the biosynthetic pathway. Chem Biol 7, 395-403.

Carmody, M., Byrne, B., Murphy, B., Breen, C., Lynch, S., Flood, E., Finnan, S. \& Caffrey, P. (2004). Analysis and manipulation of amphotericin biosynthetic genes by means of modified phage KC515 transduction techniques. Gene 343, 107-115.

Champness, W. C. \& Chater, K. F. (1994). Regulation and integration of antibiotic production and morphological differentiation in Streptomyces spp. In Regulation of Bacterial Differentiation, pp. 61-93. 
Edited by P. Piggot, C. P. Moran \& P. Youngman. Washington, DC: American Society for Microbiology.

Chater, K. F., Bruton, C. J. \& Suárez, J. E. (1981). Restriction mapping of the DNA of the Streptomyces temperate phage $\phi \mathrm{C} 31$ and its derivatives. Gene 14, 183-194.

Chen, S., Huang, X., Zhou, X., Bai, L., He, J., Jeong, K. J., Lee, S. Y. \& Deng, Z. (2003). Organizational and mutational analysis of a complete FR-008/candicidin gene cluster encoding a structurally related polyene complex. Chem Biol 10, 1065-1076.

Enríquez, L. L., Mendes, M. V., Antón, N., Tunca, S., Guerra, S. M., Martín, J. F. \& Aparicio, J. F. (2006). An efficient gene transfer system for the pimaricin producer Streptomyces natalensis. FEMS Microbiol Lett 257, 312-318.

Foussard, M., Cabantous, S., Pedelacq, J., Guillet, V., Tranier, S., Mourey, L., Birck, C. \& Samama, J. (2001). The molecular puzzle of two-component signaling cascades. Microbes Infect 3, 417-424.

Hefti, M. H., Francoijs, K.-J., de Vries, S. C., Dixon, R. \& Vervoort, J. (2004). The PAS fold. A redefinition of the PAS domain based upon structural prediction. Eur J Biochem 271, 1198-1208.

Hoch, J. A. (2000). Two-component and phosphorelay signal transduction. Curr Opin Microbiol 3, 165-170.

Huang, J., Shi, J., Molle, V., Sohlberg, B., Weaver, D., Bibb, M. J., Karoonuthaisiri, N., Lih, C., Kao, C. M. \& other authors (2005). Crossregulation among disparate antibiotic biosynthetic pathways of Streptomyces coelicolor. Mol Microbiol 58, 1276-1287.

Hutchings, M. I., Hoskisson, P. A., Chandra, G. \& Buttner, M. J. (2004). Sensing and responding to diverse extracellular signals? Analysis of the sensor kinases and response regulators of Streptomyces coelicolor A3(2). Microbiology 150, 2795-2806.

Ikeda, H., Ishikawa, J., Hanamoto, A., Shinose, M., Kikuchi, H., Shiba, T., Sakaki, Y., Hattori, M. \& Omura, S. (2003). Complete genome sequence and comparative analysis of the industrial microorganism Streptomyces avermitilis. Nat Biotechnol 21, 526-531.

Kieser, T., Bibb, M. J., Buttner, M. J., Chater, K. F. \& Hopwood, D. A. (2000). Practical Streptomyces Genetics. Norwich: John Innes Foundation.

Kim, B. S., Cropp, T. A., Beck, B. J., Sherman, D. H. \& Reynolds, K. A. (2002). Biochemical evidence for an editing role of thioesterase II in the biosynthesis of the polyketide pikromycin. J Biol Chem 277, 48028-48034.

Leskiw, B. K., Lawlor, E. J., Fernández-Abalos, J. M. \& Chater, K. F. (1991). TTA codons in some genes prevent their expression in a class of developmental, antibiotic-negative, Streptomyces mutants. Proc Natl Acad Sci U S A 88, 2461-2465.

Martín, J. F. (2004). Phosphate control of the biosynthesis of antibiotics and other secondary metabolites is mediated by the PhoR-PhoP system: an unfinished story. J Bacteriol 186, 5197-5201.

Martín, J. F., Gutiérrez, S. \& Aparicio, J. F. (2000). Secondary metabolites. In Encyclopedia of Microbiology, 2nd edn, vol. 4, pp. 213 236. Edited by J. Lederberg. San Diego: Academic Press.

Mendes, M. V., Recio, E., Fouces, R., Luiten, R., Martin, J. F. \& Aparicio, J. F. (2001). Engineered biosynthesis of novel polyenes: a pimaricin derivative produced by targeted gene disruption in Streptomyces natalensis. Chem Biol 8, 635-644.
Mendes, M. V., Antón, N., Martín, J. F. \& Aparicio, J. F. (2005). Characterization of the polyene macrolide P450 epoxidase from Streptomyces natalensis that converts deepoxypimaricin into pimaricin. Biochem J 386, 57-62.

Mendes, M. V., Tunca, S., Antón, N., Recio, E., Sola-Landa, A., Aparicio, J. F. \& Martín, J. F. (2007a). The two-component phoR-phoP system of Streptomyces natalensis: inactivation or deletion of phoP reduces the negative phosphate regulation of pimaricin biosynthesis. Metab Eng 9, 217-227.

Mendes, M. V., Recio, E., Antón, N., Guerra, S. M., Santos-Aberturas, J., Martín, J. F. \& Aparicio, J. F. (2007b). Cholesterol oxidases act as signalling proteins for the biosynthesis of the polyene macrolide pimaricin. Chem Biol 14, 279-290.

Ponting, C. P. \& Aravind, L. (1997). PAS: a multifunctional domain family comes to light. Curr Biol 7, R673-R678.

Recio, E., Colina, A., Rumbero, A., Aparicio, J. F. \& Martin, J. F. (2004). PI factor, a novel type quorum sensing inducer elicits pimaricin production in Streptomyces natalensis. J Biol Chem 279, 41586-41593.

Recio, E., Aparicio, J. F., Rumbero, A. \& Martín, J. F. (2006). Glycerol, ethylene glycol and propanediol elicit pimaricin biosynthesis in the PI-factor defective strain Streptomyces natalensis npi287 and increase polyene production in several wild-type actinomycetes. Microbiology 152, 3147-3156.

Rodicio, M. R., Bruton, C. J. \& Chater, K. F. (1985). New derivatives of the Streptomyces temperate phage $\phi \mathrm{C} 31$ useful for the cloning and functional analysis of Streptomyces DNA. Gene 34, 283-292.

Sambrook, J. \& Russell, D. W. (2001). Molecular Cloning: a Laboratory Manual, 3rd edn. Cold Spring Harbor, NY: Cold Spring Harbor Laboratory.

Sekurova, O. N., Brautaset, T., Sletta, H., Borgos, S. E. F., Jakobsen, O. M., Ellingsen, T. E., Strom, A. R., Valla, S. \& Zotchev, S. B. (2004). In vivo analysis of the regulatory genes in the nystatin biosynthetic gene cluster of Streptomyces noursei ATCC 11455 reveals their differential control over antibiotic biosynthesis. J Bacteriol 186, 1345-1354.

Sola-Landa, A., Moura, R. S. \& Martín, J. F. (2003). The two component PhoR-PhoP system controls both primary metabolism and secondary metabolite biosynthesis in Streptomyces lividans. Proc Natl Acad Sci U S A 100, 6133-6138.

Sola-Landa, A., Rodríguez-García, A., Franco-Domínguez, E. \& Martín, J. F. (2005). Binding of PhoP to promoters of phosphate regulated genes in Streptomyces coelicolor: identification of $\mathrm{PHO}$ boxes. Mol Microbiol 56, 1373-1385.

Stevens, A. M., Dolan, K. M. \& Greenberg, E. P. (1994). Synergistic binding of the Vibrio fischeri LuxR transcriptional activator domain and RNA polymerase to the lux promoter region. Proc Natl Acad Sci U S A 91, 12619-12623.

Taylor, B. L. \& Zhulin, I. B. (1999). PAS domains: internal sensors of oxygen, redox potential, and light. Microbiol Mol Biol Rev 63, 479-506.

West, A. H. \& Stock, A. M. (2001). Histidine kinases and response regulator proteins in two-component signaling systems. Trends Biochem Sci 26, 369-376.

Edited by: T. Nihira 